\title{
Esclavage, créolisation et constitution des identités en Afrique de l’Ouest : le cas des Agudàs du Bénin
}

\author{
Samuel LEMPEREUR \& João DE ATHAYDE
}

Université libre de Bruxelles ; Aix-Marseille Université

\begin{abstract}
Résumé : Les Agudàs forment une communauté aux origines afro-brésiliennes née dans le sillage de l'esclavage atlantique mais aussi domestique. Cet article montre comment l'identité de cette communauté est multiple et parfois paradoxale, et comment elle évolue en fonction du statut des personnes, de leurs histoires familiales et des conceptions de l'époque (mondialisation, revendications identitaires post-coloniales,etc.). Il met principalement en avant deux assises de cette construction identitaire : le lien avec la bourian, mascarade d'origine brésilienne, et le rapport à l'esclavage.
\end{abstract}

Mots-clés : Bénin, bourian, Agudàs, créolisation, esclavage.

Abstract: The Agudàs are a community of Afro-Brazilian origin established in the wake of both Atlantic and domestic slavery. This article shows how the identity of this community is multiple and sometimes paradoxical, and how it evolves according to people's status, their family histories, and conceptions of the time (globalisation, post-colonial identity claims, etc.). It highlights two main foundations of this identity construction: the link with the Bourian, a masquerade of Brazilian origin; and the relationship with slavery

Keywords: Benin, Bourian, Agudàs, creolisation, slavery. 
Le 13 janvier 2015, nous étions en train de mener quelques entretiens en commun à Ouidah ${ }^{1}$. En sortant du palais Houhoué, la maison du Dagbo Hounon, considéré à Ouidah comme le "chef suprême " du vodun ${ }^{2}$, nous avons salué un groupe de jeunes dont nous connaissions quelques têtes. Soudainement, parce que l'un d'entre eux nous a demandé ce que nous faisions à Ouidah, nous nous sommes retrouvés pris dans un débat sur l'esclavage et la traite atlantiques. Ce groupe de jeunes adultes (la petite vingtaine environ) était particulièrement vindicatif quant au rôle des Agudàs dans celle-ci :

- Il y a des Africains, des Ouidaniers que les Blancs ont jetés en mer, vivants

- Vous n'avez pas vu là-bas ?

- (João) Il y a eu ça ?

- Vous n'avez pas entendu l'histoire ? [...]

- C'est les Agudàs ...

- Qui ont jeté nos frères!

- Qui ont jeté les Africains dans la mer, nos frères!

- Si tu es fatigué, tu ne peux plus résister, [...] on te jetait à la mer

- (João) On te jette en mer...ah, pourquoi ?

- Mais ceux qui étaient les plus...les plus...

- Résistants, résistants!

- Qui peuvent travailler la terre, pour rester ici pour développer le continent, on les a emmenés !

Quelques instants après, ce groupe de jeunes Ouidaniers a ajouté à la responsabilité des Agudàs, celle des rois d'Abomey «venus à la rencontre des Blancs pour vendre et acheter ». Mais qui sont ces Blancs dont ils parlent ? La première exclamation ("... que les Blancs ont jetés en mer ») s'adressait aux Agudàs, une communauté aux racines à la fois brésiliennes et africaines. La seconde, désignait l'ensemble des marchands négriers occidentaux présents à Ouidah, en particulier les Français, les Anglais et les Portugais qui y avaient un fort.

Tenter de comprendre le glissement sémantique contextuel du mot "Blanc" dans les discours de ces jeunes est le sujet de cet article : les Agudàs, communauté afro-brésilienne que l'on retrouve notamment au Bénin mais aussi ailleurs sur les côtes d'Afrique de l'Ouest, sont souvent présentés (et se présentent) comme des «Blancs » malgré leur couleur de peau noire.

1 Cet article coécrit est l'aboutissement d'un travail en partie collectif et en partie individuel. Depuis le milieu du mois de décembre 2014, et pour quelques mois, nous avons décidé de profiter d'être tous deux à Ouidah lors de nos recherches dans le cadre de nos doctorats respectifs pour travailler ensemble sur certaines familles dont l'origine est dite brésilienne et dont l'histoire les lie à la traite négrière. João De Athayde est spécialisé dans l'étude de la communauté Agudà, en particulier ses rapports avec la danse et la sortie de masques de la bourian, Samuel Lempereur mène une étude socio-historique de l'esclavage domestique et de certaines généalogies familiales de l'ancien Dahomey précolonial, en particulier à Ouidah et Abomey.

2 Le vodun (ou vodoun) est le nom de la religion polythéiste locale, plus connue en français sous le nom de vaudou (et voodoo en anglais) et qui, à travers l'esclavage transatlantique, a essaimé dans les Amériques et les Antilles. Elle désigne un ensemble de croyances et de rites basé sur plusieurs divinités et forces de la nature, ainsi qu'un culte particulier aux ancêtres. 


\section{Les Agudàs : une communauté aux identités multiples}

Les Agudàs constituent une population (ou plutôt, comme nous allons le montrer, une communauté identitaire) apparue à la fin du $18^{\mathrm{e}}$ siècle, mais qui a essentiellement pris forme au $19^{\mathrm{e}}$ siècle, sur base de diverses origines sociales, ethniques et géographiques et qui, d'une manière ou d'une autre, fait référence au Brésil ${ }^{3}$.

Cette diversité des origines tient sa source dans l'organisation du commerce négrier sur les côtes d'Afrique de l'Ouest. Le projet The Trans-Atlantic Slave Trade Data $B a e^{4}$, dirigé par David Eltis, estime que le commerce entre l'ensemble du Golfe de Guinée et le Brésil seul aurait touché environ un million de captifs. Pierre Verger a montré en 1968 que la traite négrière entre l'ancienne Côte des Esclaves (entre les embouchures de la Volta et du Niger) et le Brésil s'est déroulée dans un contexte où l'essentiel des échanges humains et économiques était réalisé directement entre les deux rives de l'Atlantique. Ce commerce obéissait donc à une logique d'échanges directs sud-sud en droiture et non triangulaire comme on pourrait l'imaginer. Pour l'ensemble de la traite atlantique, la ville de Ouidah, ancien port de traite, aurait également été le lieu de passage de près d'un million d'esclaves. Ainsi, Pierre Verger (1953) a été le premier à aborder d'une manière systématique la formation d'une communauté brésilienne dans le sud du Dahomey, même si l'administrateur colonial Marty (1926) et le Brésilien Prado (1949) avaient abordé quelques-uns de ces aspects auparavant 5 .

On peut trouver chez les Agudàs principalement trois profils ${ }^{6} \mathrm{~d}^{\text {'individus et }} \mathrm{de}$ familles que nous appelons les établis, les retournés et les apparentés ${ }^{7}$. Dans la nébuleuse agudà, aux contours souvent variables ou flous, on trouve aussi les familles alliées.

Dans un premier temps, les Agudàs sont constitués pour l'essentiel de marchands du circuit de la traite négrière venus principalement de l'espace lusophone créé par le colonialisme portugais, et en particulier par le biais du Brésil (colonie portugaise jusqu'en 1822), d'où le terme de "Brésilien » qui sert d'ailleurs souvent à les désigner (Law 2013 : 44-45). Ils s'étaient établis sur l'ancienne Côte des

3 On prononce "Agoudà " et on l'écrit souvent avec cette orthographe. "Brésilien " est également une appellation emic largement répandue et, selon le contexte, plus utilisée même qu' «Agudà ». Mais puisqu'il s'agit d'introduire le lecteur aux identités présentes dans le Bénin méridional, l'utilisation du terme "Brésilien " peut prêter à confusion, d'où notre choix d' "Agudà " (De Athayde 2018 : 135-137, 141-145 et 150-153). On trouve aussi l'expression "Afro-Brésilien ", une appellation en vogue mais relativement récente. Concernant des recherches réalisées en 1995/1996, Guran (2010 : 42) remarque que l'expression commençait à peine à être introduite par les chercheurs béninois.

4 <https://hutchinscenter.fas.harvard.edu/trans-atlantic-slave-trade-database>

5 Freyre (1951) l'avait fait à la manière d'une " anthropologie de cabinet ", en reprenant les recherches de terrain envoyés par Verger qui est désigné dans l'article comme " photographe " (De Athayde $2018: 48$ 53). Quant à Prado, il « a fait très attention à ce que rapportait Pierre Verger » (Guran 2010 : 18-19).

6 Nous avons opté pour le terme de "profil ", au lieu de "groupe " qui nous semblait par trop enfermer les gens dans une catégorie fixe, ou encore de "vague ", qui avait une connotation chronologique qui ne se retrouve pas dans la réalité historique. Le terme " profil » implique de plus un caractère flou et mouvant, propre à ce qui nous semble être la sociologie et l'histoire de ces individus.

7 Parfois dits aussi les « associés". 
Esclaves pour installer des maisons de commerce, d'où le nom établis que nous leur donnons. C'est eux qui sont désignés comme "Blancs » dans l'extrait d'entretien qui introduit cet article, bien que leurs descendants soient aujourd'hui noirs de peau. Dans ce groupe, on peut citer en exemple la famille De Souza, issue du marchand brésilien Francisco Félix De Souza, le Chacha, installé sur les côtes du Dahomey aux alentours de 1800, après plusieurs voyages aller-retour au Brésil. Les De Souza, par diverses alliances, notamment un "pacte de sang " (Hazoumé 1937) entre Francisco de Souza et le roi Guézo du Danxomè, font figure de famille archétypale au sein des Agudàs établis. Ces trajectoires entre le Brésil et l'Afrique, tant physiques que sociales et familiales, $s$ 'inscrivent dans le contexte du commerce en droiture qui s'était installé entre les côtes de l'Afrique de l'Ouest et le Brésil. Cet espace luso-africain, bien moins connu que celui de l'atlantique triangulaire, est pourtant celui qui a vu transiter près de $45 \%$ des esclaves vendus à travers l'océan, soit environ 5 millions d'individus (Coquery-Vidrovitch \& Mesnard 2013). La naissance de la population agudàs est donc dès l'origine liée à des migrations entre le Brésil et l'Afrique, dans le sillage de la traite négrière, ces « flux et reflux " évoqués par Pierre Verger (1968).

Ensuite, tout au long du $19^{\mathrm{e}}$ siècle, des esclaves affranchis du Brésil arrivent régulièrement et parviennent également à s'établir sur ces mêmes côtes africaines. C'est le deuxième profil agudà, ce que l'on nomme en portugais des retornados: les retournés. A part quelques individus étant revenus en Afrique auparavant, il s'agit principalement d'une vague de centaines d'Africains, anciens esclaves affranchis, et jugés coupables (ou soupçonnés) d'avoir participé à la révolte de 1835 à Bahia. $\mathrm{La}$ " Révolte des Malés» est la plus grande rébellion d'Africains que le Brésil n'ait jamais vécue. Elle a été organisée conjointement par des Africains affranchis et des Africains esclaves, tous de confession musulmane, et présentait un fond de jihad (Reis 1995, 2003 ; Verger 1969 : 45 sq.). Cependant, bien plus importants en nombre que les Malés expulsés après la révolte, ce sont les Africains affranchis qui sont poussés à quitter le Brésil en raison d'un contexte hostile, en direction de la Côte des Esclaves, souvent avec leur famille. Celles-ci étaient pour la plupart créoles (c'est-à-dire, " nées au Brésil »), parfois métisses (mulâtres) ${ }^{8}$. Il ne s’agissait pas toujours d'expulsion à proprement parler, mais aussi d'une recherche de meilleures conditions de vie hors d'un Brésil qui avait durci ses lois et ses rapports avec les Africains affranchis (Castillo 2016). Phénomène historique et sociologique important, leur retour en Afrique a été l'occasion pour certains anciens esclaves de venir grossir les rangs de la communauté des marchands négriers qui formaient alors la première couche historique des Agudàs. Cette insertion dans la traite négrière de la part d'esclaves affranchis n'est pas limitée aux retornados, mais elle constitue l'une des particularités les plus remarquables des Agudàs. Tous ne sont pas devenus des marchands négriers, mais de par leur place au sein de la communauté agudà naissante, ils ont vécu en louant leurs services et leur savoir-faire dans les domaines les plus divers pour des trafiquants d'esclaves ou pour leurs descendants directs.

8 Sur la biographie des affranchis retournés, voir Verger 1992 ; Castillo 2011, 2016 ; Castillo \& Parés 2015, 2018. 
Le troisième profil au sein de la communauté agudà est celui des familles africaines locales issues de différentes ethnies ${ }^{9}$ qui ont rejoint la dynamique socio-économique des Agudàs, en particulier parmi les plus aisés de ces derniers. Il y a diverses raisons à cet apparentement. Les deux principales sont l'esclavage domestique et le mariage, ou à tout le moins, le concubinage. Ce sont donc des Africains qui, de gré ou de force, se sont apparentés principalement en ligne patrilinéaire aux Agudàs (quand une fille épouse un homme agudà), soit par " absorption » dans le giron des familles agudàs lorsqu'il s'agissait des descendants d'un esclave mâle qui se mettaient à porter le nom de leur maître. Les autres raisons sont notamment économiques (patronage), politiques (alliance) ou professionnelles (corporatiste). Bien entendu, ces raisons ne sont pas mutuellement exclusives et plusieurs de ces situations étaient régulièrement confondues. Dans cette catégorie d'apparentés se retrouvent donc essentiellement les descendants des esclaves domestiques africains des Agudàs et des descendants de travailleurs libres mais pauvres venus éventuellement se marier chez les Agudàs.

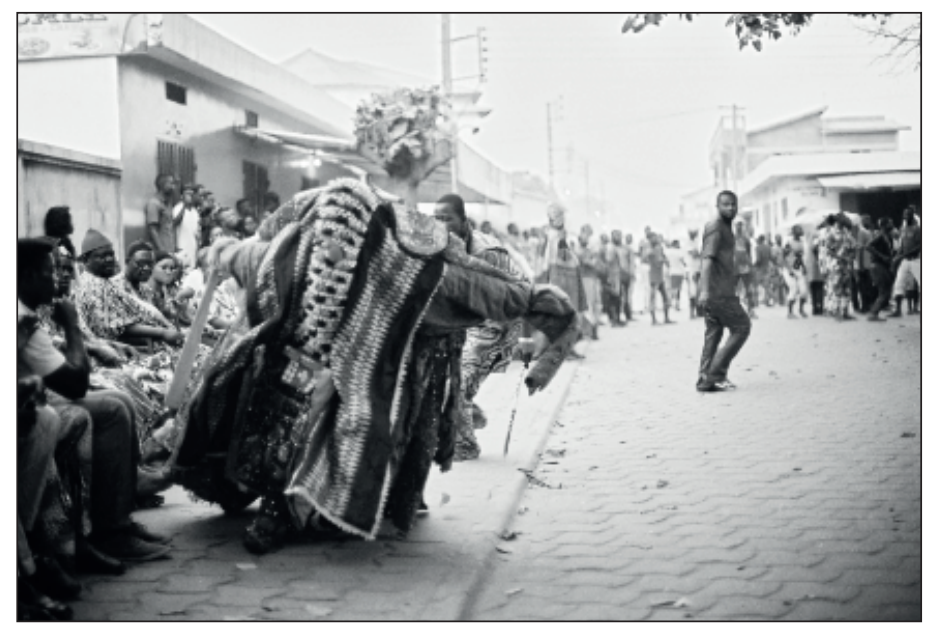

Figure 1 : Sortie de masques Egun dans une collectivité d'origine yoruba dans le centre de Ouidah. (c) Samuel Lempereur 2014.

On peut en outre distinguer un quatrième groupe, les alliés, composé des familles africaines, issues souvent de la bourgeoisie marchande locale, dont certaines sont connues encore aujourd'hui comme Adjovi, Talon ou Quenum (Houénou). Ces familles ne sont pas considérées comme agudàs mais comme des familles africaines alliées aux Agudàs. Pour des raisons d'enchevêtrements et de superpositions entre les profils que nous décrivons, qui sont plutôt à comprendre comme des idéauxtypes, certains individus et branches familiales se définissent comme agudà, alors qu'ils seraient davantage des alliés. Cela témoigne de la mise en place de diverses

9 Sur le caractère extrêmement pluriethnique et multiculturel du sud du Dahomey du Bénin méridional et de Ouidah en particulier, voir Robin Law (2013). Le mot « ethnie» s'entend ici avec toutes les précautions d'usage, étant entendu que les « ethnies ont une histoire " (Chrétien \& Prunier 1989). 
stratégies, en particulier matrimoniales ou symboliques (le prestige d'être Agudà / Brésilien). Ces familles africaines, historiquement intégrées à divers stades dans le circuit commercial atlantique (qu'il soit esclavagiste ou palmiste), appartenaient le plus souvent à l'élite précoloniale du Dahomey. Elles se sont alliées aux Agudàs avant tout par mariage, malgré une forte tendance à l'endogamie de ces derniers. Pour autant, ces alliés ne sont pas des apparentés aux Agudàs puisque s'ils le sont, c'est seulement maternellement (un homme du groupe a épousé une fille agudà). Ils ne sont dès lors généralement pas considérés comme membres de la communauté agudà puisque celle-ci est patrilinéaire à l'image du reste de la société du Sud-Bénin, bien qu'une ambiguïté existe et que certains membres de ces familles revendiquent un lien avec les Agudàs.

Ces familles alliées s'inscrivent dans la société du Dahomey/Bénin avec les Agudàs non pas sur une base culturelle, fantasmée ou non, comme c'est le cas des familles apparentées, mais plutôt sur une base sociale. Ainsi, l'étude de la place des Agudàs et de ces familles alliées d'origine africaine permet de questionner les dynamiques économiques et sociales de l'élite de l'ancien Dahomey, la stratification sociale dans son ensemble, la reproduction du capital et des élites, etc. Par exemple, ces familles africaines plus aisées envoyaient leurs enfants se faire instruire chez les Agudàs, qui avaient des écoles depuis la première moitié du $19^{\mathrm{e}}$ siècle. Ils y étaient également baptisés, et prenaient souvent le nom de leur tuteur agudà (Zinsou \& Zoumèna 2004 : 30-31). Ces échanges ont favorisé l'éclosion d'une forme de bourgeoisie locale, instruite, scolarisée et éduquée à l'occidentale. Ce type de familles alliées est un exemple parmi d'autres de la complexité des liens familiaux, sociaux, culturels et identitaires que nous étudions et montre aussi la limite conceptuelle et heuristique des catégories que nous utilisons.

Les deux profils des établis et des retournés forment le "noyau dur " des Agudàs. A celui-ci s'ajoutent de nombreuses unions ou alliances (esclaves domestiques inclus, chez les apparentés) avec des familles africaines locales (de la bourgeoisie locale, chez les alliés). Il en découle une importante influence culturelle des Agudàs sur celles-ci. Les familles du noyau dur ont effectivement un aïeul ayant vécu au Brésil ${ }^{10}$, qu'il soit marchand ou esclave affranchi. Mais ce n'est pas le cas des familles africaines locales venues s'y ajouter, que nous pouvons considérer comme des «Agudàs culturels " ${ }^{11}$. La notion d' "Agudà culturel » fournit une matrice pour comprendre l'identité agudà. En effet, au-delà des discours qui tendent à justifier l'existence de la communauté agudà par des liens de parenté, l'identité agudà se construit surtout explicitement autour de pratiques culturelles considérées comme brésiliennes (manières de s'habiller et de manger, fête de la bourian, usage de bribes du lexique portugais etc.).

L'idée d'une " communauté agudà », une communauté créole qui arrive à maintenir une cohésion identitaire assez durable, se forme au $19^{\mathrm{e}}$ siècle. Pour saisir les enjeux identitaires autour des Agudàs, il est important de garder à l'esprit qu'ils sont

10 Ou ailleurs, mais on aura compris l'importance du Brésil dans la « mythologie » ou imaginaire agudà.

11 De Athayde 2018 : 143-145. 
l'ensemble des descendants issus de toutes les interactions que nous venons de décrire et - point important - qu'en principe, ils doivent porter un patronyme à consonance luso-brésilienne, tels que Da Silva, De Souza, Do Rego, Amaral, Olympio, Da Matta, Paraïso, Sant'Ana, D’Almeida, Da Costa, etc. Cependant, d'autres individus étrangers venus de l'Occident ont rejoint aussi la dynamique des Agudàs, comme des "retournés » venus de Cuba ou de São Tomé (alors une colonie portugaise) ou même des descendants de Français ${ }^{12}$. D'autre part, certaines familles africaines qui sont restées sous la protection ou qui ont été dans l'entourage des Agudàs continuent de porter leurs patronymes africains mais sont considérées comme des Agudàs à divers degrés.

Le vocable unique "Agudà " regroupe donc une multiplicité identitaire née dans le sillage de la traite atlantique négrière. La complexité de l'identité agudà est amplifiée par un mouvement plus ou moins récent de "ré-identification ", dans lequel la volonté explicite de "retrouver ses racines " ou de "retourner à la source " devient une obsession quasi quotidienne pour certains " entrepreneurs identitaires" - ceux-ci étant entendus au sens que donnait Damien Mottier (2010) à cette expression, c'est-à-dire un ensemble d'acteurs locaux dont le projet est de définir et circonscrire l'identité agudà. Il y a aujourd'hui une forme paradoxale d'unification et d'éclatement des Agudàs. Du côté de l'unification, travaillent des associations telles que l' "Association des ressortissants brésiliens de Porto-Novo » et des associations familiales dans un sens « très étendu » comme la FRADA (Fraternité familiale D'Almeida). Du côté de l'éclatement, des individus de plusieurs familles se mettent à la recherche d'une identité familiale propre, jugée " authentique ».

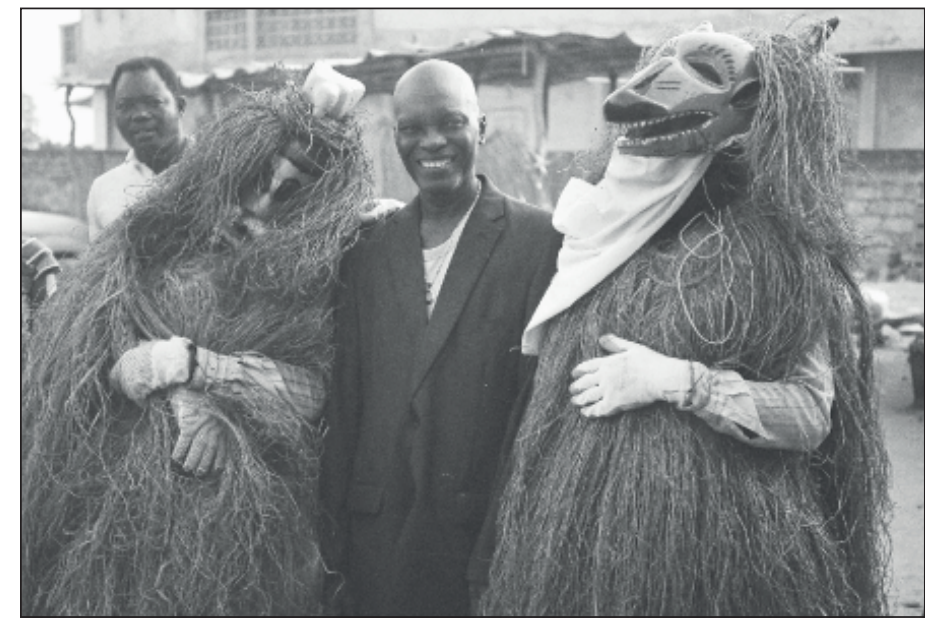

Figure 2: Sortie annuelle des Aglagodji, masques de la famille agùda Aguidissou-Da Costa à Ouidah. (c) Samuel Lempereur 2015.

12 C'est par exemple le cas des descendants de Ollivier de Montaguère, dont le nom Ollivier a été transformé en D’Oliveira pour lui donner une consonance plus lusophone. 


\section{La recherche des « racines brésiliennes " : les distinctions sociales au sein de la bourian}

La construction identitaire des Agudàs en regard avec le Brésil a été analysée de manière très brève et peu commentée par Bastide (1968) et postérieurement de manière plus consistante par Guran (2010 [1999]). Araujo (2010) a étudié comment Francisco Félix " chacha » De Souza était devenu un personnage de référence chez certains Agudàs en montrant qu'il fait figure, plus que d'un négrier, d'une sorte de bienfaiteur ayant permis à des esclaves affranchis de développer une culture proprement afro-brésilienne. Dans la famille Gomez de Ouidah par exemple, les dirigeants de la famille prennent très au sérieux la prononciation qu'ils jugent correcte de leur nom. Papa Gomez, né en 1935, retraité ayant une vie confortable dans la maison familiale à Ouidah, est parti au Portugal il y a plusieurs années à la recherche des racines de sa famille. Selon lui, il faut prononcer Gomch " à la portugaise " et non Gomez, à l'espagnole, qui serait une distorsion due aux colons français.

La construction identitaire prenant appui sur le Brésil ne se limite pas aux établis. Dans l'imaginaire des retournés, l'expérience brésilienne de l'ancêtre esclave affranchi est présentée comme une " nouvelle naissance " qui s'intègre à leur identité en se superposant avec leur attachement africain d'origine (Yoruba, Goun, Mina ...). La construction identitaire de ce noyau dur s'accompagne alors d'un phénomène de distinction sociale qui s'appuie sur leur "brésilianité ". Certains Agudàs retournés, par exemple, considèrent que les apparentés ne sont que des Agudàs " de seconde classe ". Leur " africanité " est alors souvent pointée du doigt, et on leur reproche un manque de connexion "réelle " avec le Brésil que les établis et les retournés peuvent démontrer par leur généalogie et leurs pratiques cultu(r) elles. Du point de vue des apparentés, dont beaucoup sont des descendants d'esclaves domestiques, une tendance qui nous a paru majoritaire est de se considérer pleinement comme membre de la communauté, surtout lorsque leurs familles ont conservé un patronyme à consonance portugaise ou qu'ils sont investis dans des pratiques considérées comme agudàs. Cela symbolise alors pour eux une participation culturelle à la communauté et à l'identité des Agudàs.

Les discours qui mêlent légitimité et différenciation sociale se cristallisent notamment autour de la danse bourian. Les bourians (adaptation du nom burrinha, " petite ânesse » en portugais) sont des groupes de masques réunissant une vingtaine de membres où des personnages tels que des animaux, des monstres, des humains "blancs de peau ", mais également le vodun Mami Watà dansent au rythme de la samba. Les chants se font en portugais, langue que les Agudàs ne comprennent pourtant plus depuis quelques générations.

La bourian est ainsi un étendard de la brésilianité, une manière d'évoquer en public une origine différenciée par rapport aux autres familles, considérées comme " africaines ". Cette brésilianité se mesure à plusieurs niveaux : les paroles qui font explicitement référence au Brésil, le prestige particulier des lignées historiques qui jouent la bourian parmi les établis (comme les De Souza ou, selon la version familiale, les Nevis), l'enthousiasme régulier de diverses personnalités brésiliennes 
pour la bourian tels que des ambassadeurs, des journalistes, des célébrités comme le chanteur Gilberto Gil en 1987, et même l'ancien président Lula en 2006 (De Athayde 2018). Tout cela concourt alors à légitimer certaines bourians par rapport à d'autres, mais surtout leurs responsables qui en tirent les bénéfices du prestige social. Tous les profils agudàs sont investis dans la bourian, mais à différents niveaux. L'histoire des relations entre maitres et esclaves, ainsi qu'entre lignages aînés et cadets explique notamment qu'on retrouve aujourd'hui, en général, plutôt des Agudàs établis ou retournés à la direction des principaux groupes de bourians et des apparentés parmi les musiciens et les porteurs de masques. Papa Gomez nous rappelait ses souvenirs d'enfance à ce sujet :

Pendant les repas, pendant les fêtes, les Agudàs mêmes restaient à table, et les serviteurs tapaient les tamtams, usaient les masques et dansaient et égayaient les patrons... J'étais petit, je me souviens... mais de manière très vague, parce que nous-mêmes, en ce moment on libérait déjà les esclaves, on ne leur faisait plus tellement de rigueur. Et il y avait une intégration plus grande puisque les Agudàs-mêmes épousaient des filles des serviteurs, ça s'est mélangé pour ça. (Entretien, 15 janvier 2015, Ouidah)

Puisque la bourian est un indicateur culturel de brésilianité si important pour l'identité agudà, elle est aussi un lieu propice à la distinction sociale. Karim Da Silva, riche entrepreneur à succès et personnalité bien connue de Porto-Novo dont il est l'un des leaders de la communauté musulmane, fournit une belle étude de cas à ce propos. Il est très investi dans la bourian et a été le président d'honneur ainsi que principal sponsor de l'Association des ressortissants brésiliens de Porto-Novo, non sans créer beaucoup de controverses. Karim Da Silva a aussi été le consul honoraire du Brésil, mais sur la fin son rapport avec les autorités brésiliennes s'est dégradé.

Il nous expliquait à propos de la bourian des Gonzalo, fondée en $1992^{13}$ et organisée par un groupe d'Agudàs apparentés, qu'elle n'était, en définitive, pas légitime pour plusieurs raisons.

D'abord, parce que ces nouvelles bourians trahissaient l'aspect originel de la mascarade en y introduisant un aspect financier, et en jouant non plus après cotisation des membres du groupe mais sur demande et paiement d'un tiers.

- Karim : Ça ce n'est plus pour le but culturel, c’est pour le but économique. Parce que, quand il y a les fêtes, on appelle le bourian quand quelqu'un est mort... On appelle le bourian... Ça c'est le but économique ça ; c'est pas pour la culture. La culture, on cotise l'argent ${ }^{14}$. Ce qu'ils font là, ils sont payés pour aller jouer ; c’est différent ça.

13 La bourian de Gonzalo (ou Gonzallo) a été décrite par Guran (2010 : 179, 187-189).

14 Dans les mois qui précèdent la fête du Bonfim - qui se déroule en janvier et au sein de laquelle a lieu la principale sortie bourian de l'année - le responsable pour les masques bourian, normalement accompagné par une des doyennes en charge des tissus et d'autres aspects concernant la messe et le repas, fait le tour des familles agudàs au nom de l'association de ressortissants brésiliens de Porto-Novo, pour une visite de courtoisie pendant laquelle on récolte les contributions. Plusieurs Agudàs témoignent que ces visites, ainsi que l'engagement suscité par les cotisations, soudaient les liens dans la communauté. Sur les cotisations et la circulation de l'argent autour de la bourian et la fête du Bonfim: Guran (2010:150) et De Athayde (2018: 79-81 et 290-313). 
- (João) : Et avant ce n'était pas comme ça ?

- Karim : Ah, non ! [...] Je vois qu'il y a des groupes, surtout ceux qui ne sont pas vraiment agudàs qui sont avec nous [nous soulignons], ils voient que le côté où ça peut leur apporter de l'argent. Et ils font du n'importe quoi. (Entretien, 9 juillet 2013, Porto Novo)

En désignant " ceux qui ne sont pas vraiment agudàs qui sont avec nous ", l'interviewé souligne leur déficit de légitimité : si la famille dont il est question et qui a monté un groupe de bourian n'est pas considérée comme "vraiment agudà ", c'est parce qu'elle " [est] avec nous ", une manière pudique mais néanmoins normative de désigner ces Agudàs apparentés, vus comme " trop africains » et qui ne peuvent dès lors revendiquer une origine brésilienne considérée authentique. Ils sont alors accusés de ne pas jouer la bourian pour des bonnes raisons ("C'est pas pour la culture») mais pour des mauvaises ("C’est le but économique ). Ici se lit également une distanciation sociale avec les anciens esclaves domestiques qui forment une partie majoritaire des Agudàs apparentés. Au détour de ces considérations sur le déficit de légitimité se dévoile le stigmate social lié aux descendants d'esclaves au Bénin.

- (João) : J'ai entendu parler de [la famille] Rodriguez qui faisait une bourian à Bohicon, vous la connaissez?

- Karim : Il peut en avoir un peu partout, mais les gens qui sont restés avec nos papas [nous soulignons] eux, ils le font pour avoir de l'argent, ils cherchent, ils prennent des noms agudàs; ils se font appeler comme ça pour pouvoir tromper les gens.

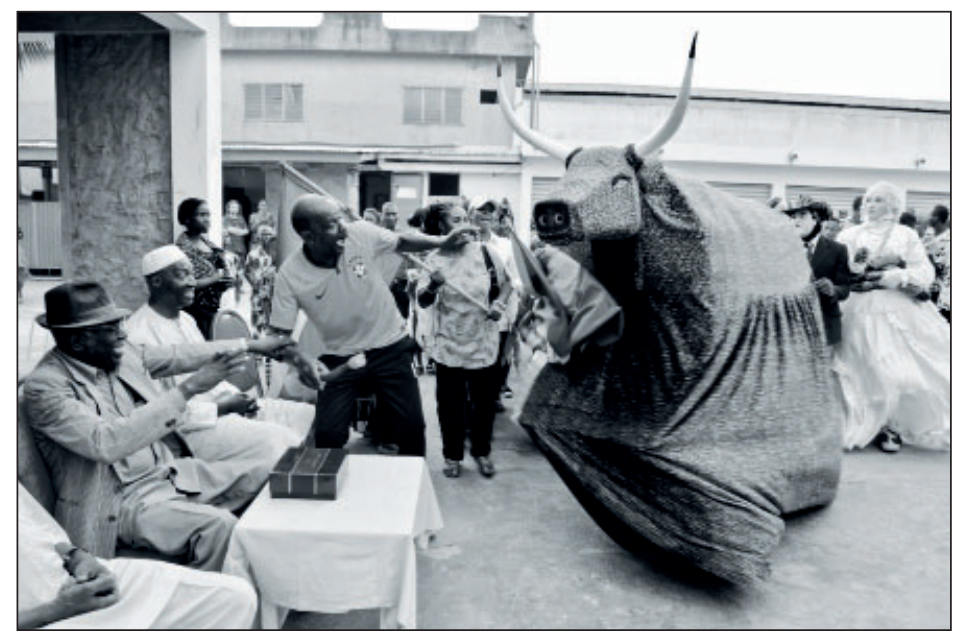

Figure 3 : Le cortège de la bourian, Porto Novo. @ João De Athayde 2015.

Après avoir parcouru la ville, avec à sa tête le chef Auguste Amaral (en maillot du Brésil), le cortège de la bourian s'arrête chez l'homme d'affaires agudà yoruba, Karim Da Silva (avec le chapeau) pour recevoir son soutien sous forme de billets qu'il garde dans une boite en carton posée devant lui.

Karim Da Silva se présente comme un descendant d'affranchi retourné, mais Guran et Reis (2002) ont montré qu'il est le descendant de José Bambeiro Paraïso, 
un esclave africain acheté à Bahia par Domingos Martins pour le servir à PortoNovo en qualité de barbier. D’identité revendiquée de descendant d'affranchis au Brésil et revenu libre en Afrique, il deviendrait ainsi le descendant d'un esclave en Afrique, ce qui lui est insupportable ${ }^{15}$.

Le discours de Karim sur l'esclavage et son passé familial qu'il tente de cacher n'entre pas en contradiction avec l'appartenance à un groupe social ayant massivement eu recours à l'esclavage domestique car "la mémoire des inégalités passées - en particulier de celles héritées de l'esclavage - est, dans certains contextes, soigneusement entretenue et susceptible d'être rappelée " (Ologoudou 2008 : 83).

Dans cette optique, il faut comprendre que "faire la bourian pour avoir de l'argent " serait une forme de trahison de l'esprit originel de la mascarade. A cette nostalgie du passé idéalisé de la bourian s'ajoute un dédain des classes aisées envers les plus pauvres qui s'est accentué ces dernières décennies quand cette danse est devenue source de revenus complémentaires pour des danseurs et chanteurs issus des couches populaires. Aujourd'hui, les pauvres se font payer pour jouer et les plus aisés paient pour se faire divertir ou entretenir la mémoire de leurs aïeux, car la bourian se joue notamment lors de funérailles et anniversaires de disparition. A ce dédain s'ajoute celui généralement porté sur les lignages cadets au sein de la parenté par les lignages considérés comme aînés ${ }^{16}$. Si tous les lignages cadets au sein de la parenté ne sont pas constitués d'anciens esclaves, loin de là, les descendants d'esclaves se trouvent bien en situation de lignages cadets. La position inférieure dans la parenté des anciens esclaves, ainsi que leur origine honteuse sont toujours susceptibles d'être rappelées. C'est à sa manière ce qu'illustre la phrase que nous avons soulignée dans l'extrait du témoignage de Karim : les " gens restés avec les papas " désigne explicitement les anciens esclaves domestiques de la famille, qui auraient "pris " des noms agudàs, une pratique courante dans une société esclavagiste, mais qui, dans l'optique de Karim Da Silva, serait alors signe de " ne pas être des Agudàs " dans le sens plein du terme, voire faite pour tromper les gens afin d'organiser des bourians et ainsi gagner de l'argent et du prestige.

15 Karim est en forte colère contre Guran à ce propos : “J'ai brûlé son livre ! " (De Athayde 2018 : 386). Signalons également pour appuyer ce besoin de s'identifier à une victime affranchie de l'esclavage atlantique que Karim Da Silva, toujours en qualité d'entrepreneur identitaire, est aussi à l'origine du "Musée Da Silva des arts et de la culture afro-brésilienne " sur les murs duquel s'étalent des bas-reliefs mettant en scène la violence de l'esclavage avec des slogans du type «L'esclavage est un crime contre l'humanité" ".

16 Les lignages cadets sont les lignages issus des enfants les plus jeunes d'un aïeul et qui, pour cette raison, ont moins de prestige et de légitimité lors de l'accès à des postes de décision, de représentation et de pouvoir au sein de la famille. Ces positions de prestige sont destinées préférentiellement aux lignages descendants des enfants les plus âgés, les lignages ainés, qui "prendraient en tutelle " les cadets. Ici, il n'est pas question donc d'être plus ou moins âgé dans le présent (ce qui entre néanmoins en jeu à un autre niveau), mais d'être le descendant d'un aïeul qui était biologiquement le cadet ou l'ainé de sa génération au moment de la création des lignages. Ainsi, est appelé " petit frère " un lointain cousin, sans considération des âges des personnes. Cela peut avoir différentes conséquences comme le choix d'un chef de famille (le plus souvent choisi dans les lignages aînés), la conduite d'un rituel (le choix du chef de culte) ou concernant des questions foncières (qui est légitime pour hériter). Avec le temps et l'assimilation, on retrouve dans les lignages cadets les descendants d'esclaves dans les enjeux identitaires liés aux Agudàs, les descendants d'établis ou de retournés tendant à considérer les apparentés dans leur ensemble comme des " cadets". 
D'autres éléments sont également mis en avant pour disqualifier cette bourian et, in fine, pour disqualifier la famille de leur qualité de "vrais Agudàs » et donc de s'en distinguer. Il s'agit alors de souligner leur manque de capital comme " agudà culturel ». En particulier, certains Agudàs du noyau dur, dont Karim Da Silva, dénoncent l'enchevêtrement de pratiques chez les Gonzalo : on ne peut pas à la fois faire la bourian et avoir dans sa maison des egungun, mascarade religieuse liée aux ancêtres Yoruba fortement présente dans le Bénin méridional à cause de la part importante de Yorubas parmi les anciens esclaves domestiques (Noret 2008, 2010). Cette bourian Gonzalo s'est éteinte après une quinzaine d'années d'existence. Deux raisons principales expliquent son arrêt : le premier coup dur dont elle a souffert a été la mort de son chanteur principal et " formateur ", Jo Gbédji (qui avait grandi parmi les De Souza), et le coup de grâce est survenu quelques années plus tard avec l'inondation qui a détruit leurs masques et accoutrements. Ces deux événements ont alors parfois été interprétés comme étant une sorte de "châtiment divin " dû au fait que la famille a voulu entretenir des eguns et un groupe bourian en même temps. Toutes ces critiques reposent sur le fait qu'ils ne pouvaient revendiquer un capital « agudá culturel » suffisant, notamment de par leur position de famille africaine apparentée $e^{17}$.

Cependant, un groupe familial apparenté peut tout à fait bénéficier de ce capital. C'est d'ailleurs le cas d'Ernest " Ninin » Kangni, le chef ${ }^{18}$ de la « Super bourian De Souza de Ouidah », l'une des troupes les plus prestigieuses du Bénin. Ernest est le fils de l'ancien chanteur de la bourian de la "Société Brésilienne Dom Francisco De Sousa », Théodore "Téko » Kangni (d'origine mina). La grand-mère paternelle de Théodore (l'arrière-grand-mère d'Ernest) était une De Souza. Les frères d'Ernest font également partie de la bourian, sa famille constitue un exemple de famille d'apparentés investie dans la bourian d'une famille tutélaire. La direction de la bourian est partagée avec Victor De Souza, qui occupe le poste de président du groupe, sans être ni musicien ni chanteur, mais qui est notamment le responsable pour la récolte d'argent offert par le public aux masques. Dans les groupes bourian, le chef est souvent aussi le président du groupe; dans le cas de la Super bourian De Souza, l'arrangement familial constitue un équilibre entre la lignée d'un savoirfaire musical "apparenté » et le membre d'une lignée paternelle qui porte, lui, le patronyme De Souza.

17 Par ailleurs, la teneur de ces critiques suggère que la bourian au sud du Bénin, au moins pour les Agudàs du «noyau dur ", prendrait l'allure d'une forme particulière d'hommage aux ancêtres avec des aspects qui suggèrent une certaine sacralité, sans pour autant être religieuse dans le sens plus strict. Pour ces Agudàs "du noyau dur ", la bourian est une pratique où l'on rend hommage aux ancêtres et qui requiert donc de la légitimité. Ceci suppose parfois un certain niveau de fidélité et d'exclusivité dédié à la mascarade, comme une forme de consécration. Ce qui les gêne le plus, semble-t-il, ce n'est pas exactement le fait que quelqu'un participe à des cultes et des sorties d'eguns, mais le fait d'être à la fois responsable d'un groupe bourian et dans le même temps d'avoir des eguns à la maison. Dans ce sens, il ne paraît pas anodin que le siège d'un groupe bourian ou le local où l'on garde les masques soit appelé " couvent " (c'est le seul terme utilisé pour cet endroit), exactement comme l'espace secret où l'on pratique le culte des eguns (et plus généralement l'ensemble du vodun). Par contre, mener un groupe bourian de manière concomitante à des fanfares ou à des groupes de musique commerciale ou traditionnelle plus profanes ne semble présenter aucun problème. Pour plus de développement sur les dimensions du sacré dans la bourian et notamment le rapport avec le culte des eguns, voir De Athayde (2018 : 397-399, 414-416, 438-439, 488-494). Le chef d'une bourian en est le chanteur, il est le responsable de la partie musicale. 
Dans ce cas, Ernest et sa lignée bénéficient donc d'un capital culturel agudá suffisant pour mériter cette place ; Ernest est d'ailleurs reconnu comme un grand chanteur dans tout le circuit des bourians entre Ouidah et Cotonou.

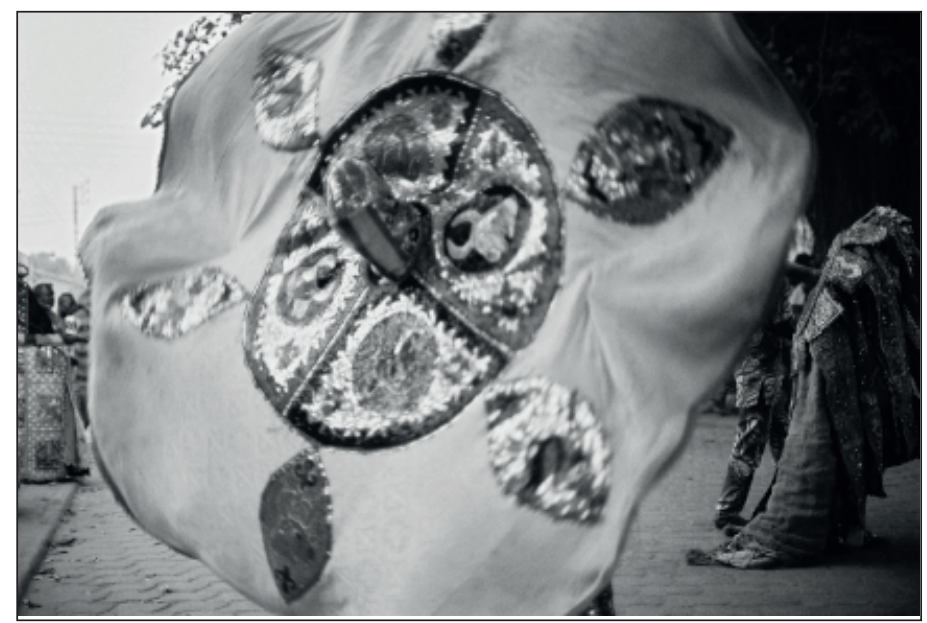

Figure 4: Sortie de masques Egun dans une collectivité d'origine yoruba dans le centre de Ouidah. (c) Samuel Lempereur 2014.

\section{Après le retour du Brésil : la quête identitaire d'une Afrique authentique}

Tous les Agudàs ne cherchent pas à s'identifier seulement comme Brésiliens, même si le Brésil reste constitutif de leur histoire. Pour certains, les racines africaines sont plus importantes. Ainsi, Lucien D’Almeida évoque les Agudás comme une communauté afro-brésilienne. Il est le descendant d'Olufade Antonio, esclave yoruba affranchi au Brésil, dont le fils Bernardino est né à Bahia le 15 juin 1830 . Ils sont revenus tous deux à Ouidah en 1840, dans le mouvement des retournés.

Les D'Almeida sont l'une des familles retornados les plus connues et leur histoire a fait l'objet de plusieurs publications (Guran 2010 : 104-106 ; Castillo 2011 ; Verger 1992 $)^{19}$. Il s'agit, dans la version officielle, de trois branches qui tirent leur nom de leur maître, Manuel Joaquim D’Almeida, capitaine du célèbre navire négrier appelé Principe de Guiné (Verger 1968 : 452).

Quand on interroge Lucien D'Almeida sur son histoire, celui-ci souligne son origine yoruba et donc africaine :

Maintenant sur le plan culturel, nous sommes Yoruba, moi je suis Nagô, [...] moi je suis d'Olufade. Je suis Nagô et on a... On a, comment dirais-je, pour pratique occulte le shango,[...] les eguns. (Entretien, 11 décembre 2015, Ouidah)

19 Verger (1992, partie V, p. 42) : "Le cas des D’Almeida est un des plus intéressants. [...] Il s'agit de trois personnes différentes : le premier, Manuel Joaquim D’Almeida est né en 1791 dans la Province brésilienne du Pernambouc, descendant de Portugais, disparu en 1854. Le deuxième, Joaquim D’Almeida, est né dans le Dahomey dans la ville de Hoko dans la région Mahi et a été durant un certain temps esclave du premier ayant reçu, par conséquent, son nom. Le troisième, Antonio D’Almeida, est né à Iseyin (actuel Nigeria), était yoruba et a été également esclave du premier, d'où le nom » [traduction des auteurs]. 
On remarque la différence avec un autre descendant de retourné comme Karim Da Silva : ce n'est pas dans la brésilianité qu'il cherche à construire son patrimoine identitaire, mais dans une origine " au Nigéria ». Cette origine se construit alors en réaction à l'esclavage, que Lucien D'Almeida qualifie de déportation :

Olufade Antonio, vous savez qu'à travers leurs noms indigènes, ils ont une source. Comme vous le savez, c'est l'esclavage... Je n'aime pas... Je n'aime pas, je parle de la dé-por-ta-tion [il insiste sur les syllabes] et non d'esclavage. Chacun est libre d'interpréter, je dis, c'est une déportation par la force... leur... on leur a imposé un nom, et ce nom, et ce prénom et dont nous sommes l'héritier aujourd'hui. (Entretien, 18 janvier 2016, Ouidah)

Quand on l'interroge sur la bourian, Lucien D'Almeida se félicite que des chercheurs brésiliens viennent rafraîchir la langue portugaise des chansons, mais précise ensuite que " en tant que Nagô ", ce n'est pas "sa chose ", mais, comme ses aïeux ont " vécu et se sont enracinés au Brésil, ils maîtrisent ".

Effectivement, tout au long du $20^{\mathrm{e}}$ siècle, les D’Almeida ont été une des familles au centre des affaires de la bourian et de la communauté agudà, notamment à Porto-Novo ${ }^{20}$. Si la place des D'Almeida comme faisant partie des Agudàs n'est pas remise en question, puisqu'il s'agit de l'une des principales et plus nombreuses familles brésiliennes du Bénin ${ }^{21}$, pour Lucien, l'identité se complète au profit d'un équilibre entre l'Afrique et le Brésil.

La mise en esclavage n'impliquant pas l'amnésie des origines (Ologoudou 2008), les Olufade Antonio D'Almeida revendiquent une histoire qui précède l'esclavage et qui aurait résisté à la mémoire de la « déportation », pour utiliser un mot important pour Lucien D’Almeida :

Olufade n'était pas tout à fait enfant [quand il est parti], c'était un adolescent, il comprenait la langue nagô et c'était un adepte de Shango ! Donc même depuis sa naissance, le Fa a déjà prédit [son retour]. [...] Quand vous êtes marqué, vous gardez ce que vous avez de plus cher. Avant son retour, il a toujours gardé le nom de ses parents, il sait d'où il vient, il sait qu'un jour il retournera. Et une fois débarqué, il l'a dit et il a cherché après des années, il a dit à son fils "Je suis d'Oyo Iseyi [Nigéria] et quand vous allez chez moi à Oyo, je suis le deuxième fils ", donc tu vois, il a gardé tout ça là de très cher. (Entretien, 18 janvier 2016, Ouidah)

A la manière de ce qu'avait montré Joël Noret (2008) dans l'investissement dans le culte egun de la part de nombreux descendants d'esclaves yoruba du Sud-Bénin, le passé d'avant la déportation permet de renverser le stigmate de l'esclavage : l'oracle Fa avait prévu son retour, il avait gardé la mémoire de ses origines et notamment

20 D’après Olinto (1964 : 183), qui a vu la bourian à Porto-Novo en 1963-1964, Casimir D’Almeida était «le patriarche de la communauté brésilienne locale». Il était le président d'honneur de la bourian et son cousin Marcelino D'Almeida l'un des principaux responsables pour la partie musicale. Casimir avait pour épouse Titia Fouliti D’Almeida, née Da Silva, tante de Karim Da Silva (De Athayde 2018 : 79, 106, 304305).

21 Comme nous l'avons vu, il s'agit des descendants de trois individus portant le nom D'Almeida sans liaison de sang entre eux (Verger 1992 : 42-54 et entretien de Lucien D’Almeida tenu à Cotonou, le 12/07/2013). De nos jours, chacun de leurs descendants connaît à quelle lignée il appartient, mais il a quand même une identification par la convergence du nom et par la référence commune à une " brésilianité », que l'on peut illustrer par la création de l’association FRADA qui cherche à réunir tous les porteurs du patronyme. 
le nom de son père Olokoku. Et surtout, il aurait été attendu par sa famille : dès la troisième génération, les Olufade D'Almeida ont entrepris de nombreux voyages initiatiques et identitaires en direction des origines d'Olufade :

Son fils Bernardino n'avait pas le culot d' [y] aller mais ses fils, ça veut dire [le] petit-fils [d'Olufade] Cosme, tout ce monde maîtrisait la langue nago dans son vrai sens, ils ont commencé par dire " Nous allons retourner " et c'est comme ça que le premier pas de retour a commencé dans le monde au Nigéria. Il n’y a que moi seul qui ne suis pas encore parti, j'y tiens, j'y tiens parce que moi aussi mon père est parti. [II] a été accueilli, [...] il est de même que nous nous allons mettre nos pas dans leurs pas, pour que l'histoire de s'efface pas. (Entretien, 18 janvier 2016, Ouidah)

On remarque l'importance des noms brésiliens dans ces premières générations : Bernardino, le fils d'Olufade né au Brésil, Cosme, première génération de retournés nés à Ouidah. A cette époque, la structuration identitaire des Agudàs passait par une endogamie préférentielle et une utilisation d'un maximum de références au Brésil. Depuis, la famille Olufade D’Almeida serait une référence, d'après Lucien D'Almeida, dans l'utilisation de la langue yoruba qui est parlée et écrite. La famille distribue autour d'elle des manuels scolaires venus du Nigéria pour servir à l'alphabétisation. La question religieuse est ici importante : d'après Lucien D'Almeida, Olufade aurait écrit dans son testament qu'il est " musulmanchrétien-animiste-laïc ». Triple appartenance religieuse que revendique également Lucien D'Almeida. Cette phrase souligne toute l'ambiguïté identitaire et religieuse de ces retournés. Les Agudàs sont associés à l'installation des premières églises au Dahomey, aux registres de baptêmes et aux fêtes catholiques en général.

Ce rapport à la religion catholique n'est alors pas sans provoquer des réactions parmi les entrepreneurs identitaires agudàs de confession musulmane qui se retrouvent notamment parmi les retournés suite à la Révolte des Malés. Ainsi, à Agoué, Nissilou Farrouk Sant'Ana, Président de l'Association des Agudàs d'Agoué, nous demandait avec malice qu'on lui explique ce que l'on entendait par "Agudà », car lui " ne sait toujours pas ce que c'est". Monsieur Nissilou Sant'Ana est plus radical dans sa quête identitaire africaine que Lucien D'Almeida, car il remet fortement en cause l'identité portugaise que certains revendiquent :

- Nissilou Sant'Ana : Parce que, je vous donne l'exemple de... de Barbosa à Agoué ici. Ils [disent qu'ils] sont plus blancs que moi. Et maintenant eux [disent qu'ils] ne sont pas des Africains, [qu'] ils sont des Portugais. Ça ne va pas dans votre tête ? On en trouve plein-là qui... qui... qui répètent les mêmes choses.

- (Samuel) : On entend souvent quand on va dans les familles agudàs, ils disent souvent qu'ils sont yovo... Ils disent qu'ils sont blancs.

- Nissilou Sant'Ana : Oh non, non! Que leur père est blanc ?

- (Samuel) : Parfois un arrière-grand-père...

- Nissilou Sant'Ana : Un arrière-arrière maître qui a acheté... Là... On te prend... Bon, allons-y. Parce que la traite là, on vous prend, on vous vend, d'accord.[...] On vous baptise de force. Quand vous arrivez là-bas, celui qui vous achète, il vous baptise [...] et vous prenez son nom ! 
[...] C'est ça qu'il s'est passé, c'est ça que les gens doivent savoir. [Que] quelqu'un vienne me dire que lui, il est agudà, il est blanc, [...] je vais dire "Vous vous foutez de qui ? " Hein ? Si je te sors... le... les statuts et règlements intérieurs des Agudàs, je les ai lus, j'ai dit " Non, je ne suis pas d'accord avec tout ». (Entretien, 28 janvier 2016, Agoué)

Les deux exemples de Lucien D'Almeida et Nissilou Sant'Ana montrent que l'identité agudà est traversée de tensions, et l'imaginaire du Brésil y tient une place importante, notamment chez les personnes au sein de mouvements politiques de type afro-centriste ou panafricain, comme c'est le cas pour Lucien D'Almeida et Nissilou Farouk Sant'Ana ${ }^{22}$.

La revendication d'une culture proprement africaine n'est pas l'apanage des retournés, ce qui aurait pu alors se lire comme l'expression de la tension identitaire induite par la double traversée de l'Atlantique d'un aïeul. L'ambivalence entre les revendications identitaires multiples se retrouve également, à des niveaux d'intensité différents selon les cas, chez les apparentés. Emile Ologoudou, dans son article publié en 2008 dans la revue Gradhiva, souligne la volonté explicite chez son père de s'écarter de l'influence des anciens maîtres De Souza, en particulier parce que son grand-père était un devin yoruba reconnu dans la région. Emile Ologoudou est d'ailleurs toujours considéré comme un "dignitaire", dans le français local, du culte yoruba d'oro ${ }^{23}$. Dans ce cas comme dans bien d'autres, l'histoire, ou plutôt la manière de la présenter, est un enjeu qui varie selon l'interlocuteur, sa position familiale, sa position sociale, ses points de vue, ... A l'heure où le vodun est considéré comme un patrimoine à mettre en valeur, il n'est pas étonnant de voir de telles pratiques, notamment chez les plus lettrés (Ciarcia 2016a). Se présenter et décliner son identité ainsi que son passé face à des étrangers peut alors devenir complexe et créer des tensions au sein de la famille.

En avril 2016, une enquête sur les descendants d'esclaves yorubas dans les villages aux alentours de Ouidah nous a menés dans la famille Bentho, notamment réputée pour ses connaissances sur egun. Nous savions par d'autres personnes que les Bentho avaient été au service de certains Agudàs de Ouidah. Cette famille correspond donc au profil apparenté que nous avons décrit, bien qu'ici, comme c'est souvent le cas lorsque les distances sont importantes, les liens qui unissaient les lignées et les familles se sont largement distendus avec le temps. La discussion quant à l'histoire familiale a réuni Ololode Bentho, le chef de famille très âgé, et trois de ses fils présents dans la maison ce matin-là. Elle a provoqué un échange animé entre le père et ses fils quant à l'origine de la famille :

- (Samuel) : Bentho, c'est une famille fo ?

- Un des fils Bentho : Bentho est agudà. Bentho, c'est agudà.

- (Samuel) : Agudà de quelle origine?

22 Lucien d'Almeida est très actif auprès de la Marche du repentir, organisée chaque année à Ouidah (Ciarcia 2016a). A noter que tous deux revendiquent un passé en Egypte, mythologie commune de la mouvance afrocentriste.

23 Oro est un culte yoruba lié aux ancêtres. Il se manifeste la nuit, est secret et strictement réservé aux hommes. 
Mais un autre fils nous a interrompu et s'est écrié en fongbe :

Aguda yovo e! Aguda yovo e! Bentho yovo e a!

(Les Agudàs sont des Blancs! Les Agudàs sont des Blancs! Les Bentho ne sont pas des Blancs!)

On devine ici l'ambivalence des liens avec leurs anciens maîtres auxquels la famille Bentho avait été rattachée. Fallait-il se dire agudà ? Ou yoruba ? Le premier fils à avoir répondu sur leur origine avait spontanément dit "agudà ", et l'un de ses frères l'avait corrigé. Diminué par l'âge, Ololode Bentho avait du mal à répondre à ses fils qui débattaient entre eux, discussion dans laquelle s'était ajouté notre interprète ${ }^{24}$ qui, de bonne volonté, tentait de les raisonner en leur expliquant qu'ils ne pouvaient être à la fois yorubas et brésiliens. Cette ambiguïté n’a jamais disparu des entretiens menés dans cette famille, au point de revendiquer " être catholiques quand on est à Ouidah ", dans une famille - nous le disons - reconnue pour ses connaissances dans les cultes yorubas. Signe, s'il fallait le souligner, du patronage encore relativement important des familles agudàs sur leurs anciens esclaves.

\section{Blancs, Noirs, Créoles : une identité pigmentaire entre biologie et construction sociale}

A l'époque précoloniale, les Agudàs faisaient partie de l'élite commerciale et politique, leurs manières culturelles et sociales étaient différentes de celles des Africains. Les premiers Agudàs établis étaient des Occidentaux : ils venaient du Brésil, du Portugal, de France, de Cuba ...

C'est dans ce contexte qu'ils sont historiquement qualifiés de "blancs " ${ }^{25}$. Le terme est resté avec le métissage au fil des générations, en désignant les créoles brésiliens ou portugais et qui, selon une espèce de "One drop rule » locale, se recrutent loin dans la parenté ${ }^{26}$. Le colonialisme, à l'image de ce que Stoler (2002) a souligné, participe à renforcer l'effet prestigieux de la créolité, autant parce que les Agudàs seront recrutés massivement comme intermédiaires locaux du colonialisme que parce qu'ils représentaient «la civilisation ${ }^{27}$. Plus que leur couleur de peau, cette attribution pigmentaire désigne donc une manière de vivre qui renvoie à une forme de stratification sociale ${ }^{28}$. C'est bien l'ensemble des pratiques sociales et culturelles

24 Il s'agit de Hugue Akowhendo, jeune instituteur, également descendant d'un esclave yoruba et d'une famille parente aux Bentho.

25 Certains étaient même peut-être déjà métissés ; c’est le cas de Francisco De Souza, né à Salvador de Bahia et qui, selon la tradition familiale, serait le fruit de l'union entre un Portugais et une Amérindienne (Silva 2004 : 12 ; De Souza 1992 : 23). A propos des retournés venus de Cuba et qui s'intègrent à une dynamique agudà-brésilienne, voir Sarracino 1988 et Otero 2006. Ce phénomène est, toutefois, plus visible au Nigéria qu'au Bénin.

26 A ce propos, voir la notion de One white drop rule (De Athayde 2018 : 148-150).

27 Pour reprendre une expression d'Emile Ologoudou lors d'un entretien en janvier 2016. En contrepoint, nous pouvons citer un autre intellectuel béninois, Paul Hazoumé, repris dans l'ouvrage de Zinsou \& Zoumènou : "Pour eux, nous étions des arriérés, des sauvages » (2004 : 30).

28 Cependant, l'assignation à la couleur de peau n'est jamais loin. Ainsi, Lionel Zinsou, ancien Premier ministre béninois et candidat malheureux aux élections présidentielles de 2016, est parfois considéré, en toute bonne foi, par certains Béninois (en particulier dans le contexte du sud du Bénin) comme Agudà, à la fois pour ses manières occidentales ainsi que son statut social et milieu d'origine, que pour le fait 
d'un groupe métissé qui font de cette "identité blanche » une attribution sociale et culturelle plutôt que " raciale » ou "biologique " $^{29}$. C'est, d'une certaine manière, ce que souligne Robin Law quand il écrit quà Ouidah, une ville construite sur un mélange social depuis plusieurs siècles, « race » ou " ethnie » renvoient fortement à des groupes sociaux (Law 2013: 52-53). Au cœur d'une ville fortement multiethnique où les identités religieuses, sociales et culturelles sont déjà multiples, la communauté agudà est à l'image d'une poupée russe identitaire : un groupe social multi-identitaire dans un milieu social qui l'est tout autant.

La fin des années 1980 est alors un tournant pour la communauté. Elle débouche sur un questionnement de l'identité qui s'exprime à travers les exemples que nous avons choisis. La fin du régime marxiste-léniniste de Kerekou ouvre à nouveau la voie aux familles agudàs, en particulier aux familles De Souza et Vieyra. Le cardinal Isidore De Souza est à la tête de la Conférence nationale qui conduit la transition et Rosine Vieyra, épouse du président Nicéphore Soglo, est députée et l'une des figures de proue du parti de son mari. Son frère, Désiré Vieyra est ministre et l'homme fort du gouvernement Soglo (Tozzo 2004 ; Guran 2010 : 45; Strandsbjerg 2015).

C'est à la suite de ce " renouveau démocratique " que - en particulier à Ouidah - plusieurs événements sont mis sur pied pour tenter de commémorer la traite atlantique, à leur manière : Ouidah 92, Porte du Non-Retour, Route de l'Esclave, ... Parce que ces événements réveillent " des souvenirs enfouis ", ils ont alors pour " effet social » de permettre de venir « interroger et scruter des mémoires jusqu'alors silencieuses, défaillantes ou rétives "(Ciarcia 2013: 89). Dans ce contexte d'énonciation de l'esclavage domestique avec tout ce qu'il suppose comme intimité familiale à dévoiler, le vodun mondialisé par l'effet de la traite prend alors une "dimension œcuménique » entre l'Afrique et le reste du monde et devient un symbole d'africanité authentique. Le "stigmate de l'esclavage comme emblème " (Ciarcia 2016b) se construit dès lors aussi en fonction du contexte international. Ce dernier, suite aux injonctions d'organisations telles que l'UNESCO, ou de la diaspora africaine, qui insistent particulièrement sur la repentance par rapport à la traite atlantique, oblitère dans le même mouvement l'esclavage domestique et ses conséquences. La mondialisation des débats et des revendications politiques - et en particulier celles qui touchent à la question identitaire postcoloniale produit aussi ses effets parmi les élites urbaines de Ouidah, et les tensions autour des identités à revendiquer se laissent voir au gré de la manière dont les Agudàs, qui font office de locomotives identitaires, veulent se présenter.

qu'il est métis et a un teint de peau clair. En guise d'exemple, dans le Historical Dictionary of Benin (Hougnikpo \& Decalo 2013), on trouve en page 6: "As a member of the 'Brazilian' elite, Zinsou had neither an ethnic nor a regional power base".

29 Il faut souligner que de nos jours, "yovo " (" blanc " en fongbe) sert toujours pour une partie de la population plutôt rurale et /ou populaire à désigner les Béninois expatriés (même temporairement pour des études), ou plus encore à désigner la bourgeoisie urbaine qui utilise plus facilement le français que le fongbe, ou dont les manières socio-culturelles « rappellent » celles des Blancs. 
Des auteurs ont souligné récemment que, paradoxalement, les discours des institutions internationales sur l'esclavage ne jettent pas complètement l'opprobre sur une famille de négriers comme les De Souza parce qu’ils permettent également de mettre en avant un passé glorieux, le métissage et le brassage des cultures qu'a permis la traite atlantique (Araujo 2010 : 128 ; Ciarcia 2016a, 2016b).

Mais chez les retournés, ou les apparentés, la question est tout aussi ambiguë et va dépendre notamment de l'interprétation de la place de leurs ancêtres dans la traite négrière et la manière dont ils se projettent dans leurs propres généalogies. Cellesci deviennent alors l'une des clefs de lecture à prendre en compte pour étudier les reconfigurations identitaires. Ces tensions dépassent les profils que nous avons définis en introduction, raison pour laquelle nous n'avons pas voulu utiliser le terme " groupe » qui nous semblait par trop induire l'idée d'une catégorie fermée. Les quêtes identitaires ne se réduisent pas aux trajectoires historiques des familles et des lignées (établis, retournés ou apparentés), elles vont aussi dépendre des profils sociaux et des volontés des entrepreneurs identitaires quant à ce qu'ils entendent promouvoir. L'identité agudà n'est donc pas seulement multiple, elle est aussi et surtout profondément ambivalente en ce qu'elle se compose à la fois d'anciens maîtres et esclaves, d'anciens Occidentaux et Africains, d'anciens Africains ayant traversé l'Atlantique et d'autres dont les aïeux n'ont jamais quitté le continent, d'animistes, de catholiques, de musulmans (voire des trois à la fois dans un grand bricolage religieux), de Noirs qu'on appelle Blancs ...

C'est ainsi qu'il faut comprendre le dialogue - entre nous et les jeunes de Ouidah qui ouvre cet article : les Agudàs sont très régulièrement considérés comme des «Blancs ». C'est également face à ces ambivalences qui mélangent couleur de peau, appartenances ethnique et sociale qu'il faut comprendre les rapports à l'histoire des trois "locomotives identitaires " présentées ici. L'énervement de monsieur Sant'Ana face aux Agudàs qui se disent blancs alors que leur source est en Afrique. L'importance de la culture yoruba chez Lucien D'Almeida. La volonté d'un Karim Da Silva de se présenter comme le descendant d'un affranchi au Brésil plutôt que d'un esclave au Brésil racheté par un négrier brésilien et installé à Porto-Novo : le stigmate associé à l'esclave victime de la traite atlantique puis affranchi dans les Amériques n'est pas le même que celui d'un esclave en terres africaines. Le premier n'a plus de maître à proximité et fait partie du noyau dur d'une communauté d'élites qui a structuré le pays durant plusieurs générations, le second fréquente encore les descendants des maîtres de ses aïeux, est issu de lignages cadets et peut encore se faire rappeler régulièrement son passé. 


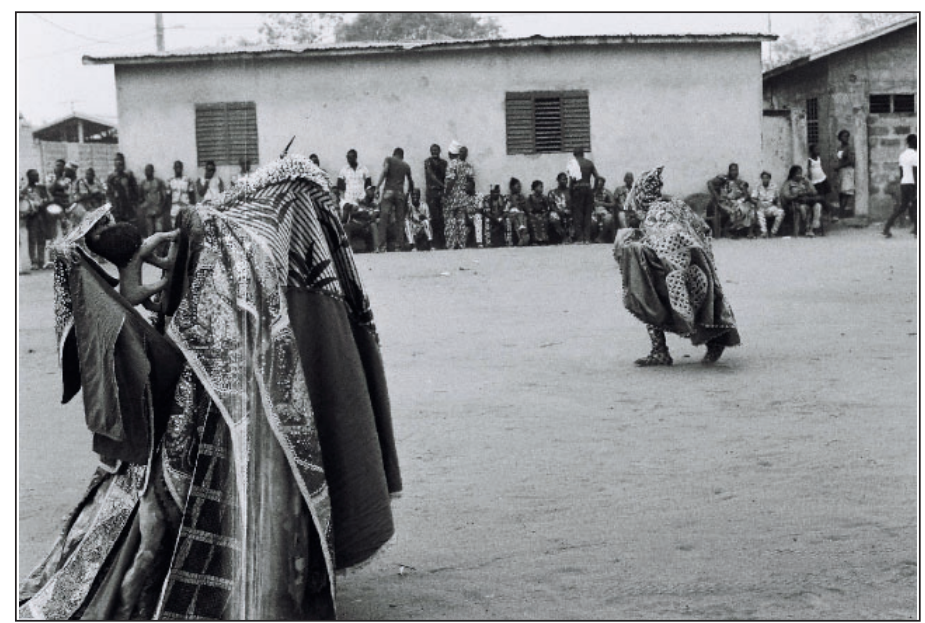

Figure 5 : Sortie de masques Egun dans une collectivité d'origine yoruba, dans les environs de Ouidah. () Samuel Lempereur 2015.

\section{Conclusion : l’identité agudà et le paradoxe de la « double conscience diasporique ॥}

On trouve chez les Agudàs une sorte de " double conscience diasporique ». Les termes de "double conscience " et de " diaspora " étaient des notions fondamentales dans la pensée de W.E.B. Du Bois (1903). Avec l'idée de "double conscience ", Du Bois essayait de théoriser la double appartenance des Noirs américains : à la fois membres de la société américaine et membre d'une " race historique " noire. Toutefois, cette dualité chez Du Bois concernait les Noirs des Etats-Unis dans un contexte ségrégationniste et de revendication des droits civils, en parlant de la communauté agudàs, ces termes doivent prendre une autre dimension.

Paul Gilroy (1993) a développé et actualisé la notion de Du Bois. Son idée de Black Atlantic incarne la "double conscience " dans la modernité, le fil conducteur de l'ouvrage est d'insister sur l'idée que l'esclavage atlantique a produit une contreculture moderne noire hybride, dynamique, qui n'est pas figée. Nous pouvons dire que Gilroy s'aligne à sa manière du côté de ceux qui, comme Roger Bastide, soutiennent qu'il y eut et il y a chez les descendants d'Africains dans les Amériques un processus de re-création ethnico-culturelle et qu'il s'agit, en fin de compte, d'une forme de créolisation.

Toutefois, nous pouvons, avec Ana Lucia Araujo, remarquer que Gilroy limite malheureusement son idée d' "Atlantique noir " à l'histoire des Etats-Unis ; il oublie l'Amérique latine et l'Afrique et « écarte alors la diversité et les inégalités qui caractérisent cette zone géographique et conceptuelle»(Araujo $2010: 2$ ) ${ }^{\mathbf{3 0}}$.

30 Traduction des auteurs. 
Il faut reconnaître que les phénomènes de créolité sont encore assez mal connus sur le continent africain ${ }^{31}$. A ce titre, le phénomène des retournés en Afrique serait ainsi une des expressions les plus remarquables de cet «Atlantique noir ", à condition de ne plus le limiter à l'Amérique du Nord. Les ancêtres des Agudàs qui ont «pris le bateau deux fois " et dont les descendants vivent en Afrique l'incarnation de cette " créolité ", vivent cette identité in between portée à son paroxysme. Toutefois, cet "Atlantique noir " où les Agudàs se placent, n'est pas seulement " racial et ethnique ", il se trouve surtout dans des articulations sociales, historiques, culturelles et généalogiques. Il faut aussi prendre garde de ne pas tomber dans ce que Chivallon dénonçait comme une aporie de Gilroy, le " pastiche de l'hybridité culturelle » (2008: 359) qui empêcherait dès lors de penser aussi les reproductions sociales et culturelles, mais également la stratification sociale interne à ces cultures dites " hybrides ». Au Bénin, les discours agudàs mettant en avant la créolisation et l'hybridité sont surtout le fruit de l'élite cosmopolite locale, comme le souligne Kadya Tall (2013) en parlant de l'ouvrage d'Araujo.

Dès lors, que serait la « double conscience diasporique » des Agudàs et en quoi estelle paradoxale? Dans la question qui nous occupe ici, l'esclavage atlantique mais aussi domestique sur le continent africain joue un rôle important. La " première conscience " n'est pas exclusive aux Agudàs, mais diffusée parmi les Béninois en général, notamment à travers la vision œecuménique du vodun que nous évoquions et son lien avec la traite. Il s'agit de la notion assez diffuse de faire partie d'une culture diasporique africaine ou négro-africaine, la conscience que la population et les identités auxquelles l'individu s'identifie ayant été transférées vers d'autres continents et constituant donc la base d'une diaspora. Les Agudàs, comme l'ensemble des Béninois, voient la "diaspora africaine " à l'écran ou en visite au Bénin et l'entendent à la radio. Même lorsque les Agudàs se réclament d'une origine brésilo-occidentale, ils se sentent en lien avec l'Afrique en tant que "signifiant flottant " à " géométrie variable " (Amselle 2001 : 15). La " seconde conscience " est celle du retour en Afrique, à laquelle se mêle une identité dès lors qualifiée de "brésilienne ", où l'on évoque une certaine "blanchitude ". Ces consciences se superposent et les Agudàs forment un groupe avec des caractéristiques identitaires assez particulières, ils sont, pourrait-on dire, une diaspora africaine en Afrique.

La communauté agudà pourrait ainsi être un exemple, hors contexte nationaliste, de la "communauté imaginaire " de Benedict Anderson ${ }^{32}$. Construite à cheval sur plusieurs continents, elle est un groupe d'appartenances multiples (parfois fantasmées ou imaginées) à caractère non-exclusif, puisque se superposent souvent l'origine brésilienne et une origine ethnique africaine (Mahi, Gun, Kpeda, Yoruba, Mina, ...), auxquelles s'ajoute encore un pluralisme religieux et social. Elle se constitue sur base de ces différentes appartenances sociales. Si la communauté agudà ne peut pas être qualifiée d'ethnie, on ne peut qu'y reconnaître plusieurs

31 Et reconnaître aussi, même si cela ressemble de plus en plus à un truisme de nos jours, que l'anthropologie, qui n'a pas toujours considéré les dynamiques sociales et historiques, a sa part de responsabilité dans cette méconnaissance.

32 Au risque de tomber dans les dérives pointées par Christine Chivallon (2007). 
des caractéristiques qu'avait mises en avant Barth sur les frontières des groupes ethniques (1995). Elle n'est pas une identité figée, et ses frontières peuvent toujours être renégociées, non seulement parce que cette communauté est bien trop hétérogène, mais car elle est également le fruit d'une historicité, en particulier depuis que le contexte international vient défier son caractère protéiforme depuis près de trois décennies. Jamais figée, elle est donc le fruit de différentes stratégies identitaires (Bayart 1996) qui se construisent en passant d'une justification à une autre (Boltanski \& Thevenot 1991).

Il faut enfin conclure sur la place de l'esclavage dans cette quête identitaire dont on peut distinguer deux moments principaux. D'abord l'esclavage liée à la traite atlantique. Il est question ici de deux différents groupes sociaux historiques qui ont fini par converger dans la revendication d'une identité commune. Les premiers sont les négriers installés sur les côtes d'Afrique, que nous avons appelé les établis. Les seconds sont les retournés, victimes des négriers qui les ont envoyés essentiellement vers le Brésil en tant que captifs. Les deux groupes finirent pourtant par constituer, au Bénin, une identité commune. Ce phénomène constitue ce qui nous identifions comme un premier paradoxe au sujet de l'identité agudà.

La seconde place de l'esclavage se situe alors uniquement en Afrique, dans un cadre appelé communément « domestique » pour le différencier de l'atlantique, ce qui ne signifie pas, pour autant, qu'il se pratiquait uniquement dans le domus, la maison, comme le montre l'usage intensif de main-d'œuvre servile dans les immenses plantations de palmiers à huile (Law 1995, 2004). Cet esclavage domestique africain souligne alors un second paradoxe. Non seulement les établis, négriers transatlantiques de métier, furent également propriétaires d'esclaves en Afrique, mais ce fut également le cas des retournés, pourtant eux-mêmes esclaves affranchis. Ce sont les descendants de ces esclaves domestiques qui forment le plus gros du nombre des individus qualifiés ici d'apparentés. L'esclavage est donc au centre des multiples reconfigurations identitaires agudàs, bien que ce n'en soit pas la seule matrice. En effet, nous avons vu que les considérations socio-économiques, de distinctions sociales et de pratiques culturelles doivent aussi être prises en compte.

Mais l'esclavage est aussi au centre des considérations familiales. On ne peut donc pas simplement dire que l'esclavage est "l'antithèse de la parenté " (Meillassoux 1986 : 35), ou qu'il est " antinomique " à la parenté (ibid. : 318). Sans refaire le débat ${ }^{33}$, il ne suffit pas pour autant de proclamer avec Miers \& Kopytoff (1977) qu'il se situe dans le continuum de la parenté. A ce titre, les allers-retours et ambivalence de l'exemple présenté ici des Agudàs quant à l'esclavage suggèrent deux choses.

La première est que Meillassoux et Miers-Kopytoff ne sont peut-être pas autant mutuellement exclusifs qu'on le laisse parfois entendre. Les esclaves ont bien souvent une origine hors parenté ${ }^{34}$, mais ils peuvent tout à fait y être intégrés en une ou deux générations soit par des pratiques matrimoniales, soit par le prisme

33 Dont Jean-Pierre Warnier (1988) a fourni un résumé à l'époque.

34 Nous mettons ici volontairement de côté les très rares cas d'esclavage au sein d'une même parenté dont Charles Piot fournit un exemple (Piot 1996). 
d'une parenté " rhétorique " (qui, le plus souvent, ne peut être dévoilée qu'au prix d'une généalogie poussée). Cependant, c'est une parenté un peu particulière, que le terme de continuum ne permet pas de parfaitement recouvrir, et qui est toujours potentiellement entachée d'un stigmate (le rappel à l'origine servile). La relation esclavage-parenté est donc ambiguë et s'exprime dans l'ambivalence de l'identité agudà, par exemple quand certains dénigrent les apparentés comme des «mauvais» membres de la communauté.

La seconde est la mise en lumière d'une sorte de " généalogie de la morale " esclavagiste. L'ambiguïté autour du rôle des retournés, et donc de leur faute, au sens où ils sont à la fois victimes puis perpétrateurs de l'esclavage. Lucien D'Almeida, dont l'aïeul a eu des esclaves domestiques - comme en témoigne son testament - insiste donc sur l'horreur, non pas de l'esclavage, mais de ce qu'il appelle la "déportation ». C'est donc le fait d'avoir réduit des Africains en esclavage pour un autre continent qui est vu comme le mal, mais aussi le début du besoin d'une quête identitaire qui est à chercher dans les origines yorubas de la famille.

Le paradoxe de la double conscience diasporique se retrouve alors renforcé par le paradoxe induit par l'esclavage, qui est alors à la base de l'histoire et des constructions identitaires multiples agudàs, tout en étant à la fois un repoussoir auquel on va tenter de ne pas être assimilé.

\section{Remerciements}

Ce travail n'aurait pas été possible sans l'apport de nombreuses personnes, collègues et amis dont la liste exhaustive serait impossible à dresser. Nous tenons cependant en particulier à remercier les Béninois qui nous ont accordé du temps, de l'énergie et de la confiance, en particulier les familles D’Almeida, De Souza, Kangni, Da Silva, Nevis, Gomez, Sant'Ana, Bentho et Ologoudou qui apparaissent dans cet article.

\section{Références citées}

Amselle, Jean-Loup, 2001. Branchements. Anthropologie de l'universalité des cultures. Paris: Flammarion.

Araujo, Ana Lucia, 2010. Public memory of slavery. Victims and perpetrators in the South Atlantic. Amherst/New York : Cambia Press.

Barth, Fredrik, 1995. «Les groupes ethniques et leurs frontières », in Philippe Poutignat \& Jocelyne Streiff-Fénart (éds), Théories de l'ethnicité, pp. 205-250. Paris : PUF.

Bayart, Jean-François, 1996. L'illusion identitaire. Paris : Fayard.

Castillo, Lisa Earl, 2011. "The exodus of 1835 Àguda life stories and social networks ", in Tunde Babawale et al. (éds), Panafricanism and the integration of Continental Africa and Diaspora Africa, pp. 27-51. Lagos : Centre for Black and African Arts and Civilization .

Castillo, Lisa Earl, 2016. "Mapping the nineteenth-century Brazilian returnee movement : Demographics, life stories and the question of slavery ,Atlantic studies 13 (1), pp. 25-52.

Chrétien, Jean-Pierre \& Gérard Prunier (dir.), 1989. Les ethnies ont une histoire. Paris : Karthala.

Ciarcia, Gaetano, 2013. «L'oubli et le retour », L'Homme 206, pp. 89-119. 


\section{Samuel LEMPEREUR \& João DE ATHAYDE}

Ciarcia, Gaetano, 2016 a. Le Revers de l'oubli : mémoires et commémorations de l'esclavage au Bénin. Paris : Karthala/Ciresc.

Ciarcia, Gaetano, 2016 b. « Du stigmate comme emblème : le cumul des mémoires à Ouidah, ancien comptoir négrier », Ethnologie française 164 (4), pp. 691-700.

Chivallon, Christine, 2007. « Retour sur la 'communauté imaginée' d’Anderson : essai de clarification théorique d'une notion restée floue », Raisons politiques 27 (3), pp. 131-172.

Chivallon, Christine, 2008. "Black Atlantic revisited : une relecture de Paul Gilroy pour quelques prolongements vers le jazz », L'Homme 187-188 (3), pp. 343-374.

Da Costa e Silva, Alberto, 2004. Francisco Félix de Souza, mercador de escravos. Rio de Janeiro : Nova Fronteira/EdUERJ.

De Athayde, Joao, 2018. "Bourian ou la danse des maîtres. Circulations et enjeux identitaires des Agudàs, les Brésiliens du Bénin », thèse de doctorat en anthropologie, Aix-Marseille Université.

Du Bois, William Edward Burghardt, 1903. The souls of black folk : Essays and sketches. Chicago : A.C. McClurg \& Co.

Gilroy, Paul, 2007. The Black Atlantic : Modernity and double consciousness. Londres : Verso.

Guran, Milton, 2010 [1999]. Agoudas, les «Brésiliens » du Bénin. Paris : La dispute.

Hazoume, Paul, 1937. Le pacte de sang au Dahomey. Paris : Institut d'ethnologie.

Houngnikpo, Mathurin \& Samuel Decalo, 2013. Historical Dictionary of Benin (4 $4^{\text {th }}$ edition). Lanham, Toronto, Plymouth (UK) : The Scarecrow press.

Kadya Tall, Emmanuelle, 2013. "Araujo, Ana Lucia. Public memory of slavery », Cahiers d'études africaines 202, pp. 935-938.

Law, Robin (ed.), 1995. From slave trade to "Legitimate" Commerce. Cambridge : Cambridge University Press.

Law, Robin, 2004. Ouidah : The social history of a West African slaving 'port' 1727-1892. Athens : Ohio University Press.

Law, Robin, 2013. « Ouidah as a multiethnic comunity », in Jorge Cañizarez-Esguerra, Matt D. Childs \& James Sidbury (éds), The black urban Atlantic in the age of the slave trade, pp. 42-62. Philadelphia : University of Pennsylvania Press.

Marty, Paul, 1926. Etudes sur l'islam au Dahomey. Paris : E. Leroux.

Meillassoux, Claude, 1986. Anthropologie de l'esclavage. Paris : Presses universitaires de France.

Miers, Suzanne \& Igor Kopytoff (éds), 1977. Slavery in Africa. Madison : The University of Wisconsin Press.

Mottier, Damien, 2010. «Entreprenariat charismatique et réveil prophétique. Creuset congolais et prophétisme ivoirien en France ", in Sandra Fancello \& André Mary (éds.), Chrétiens africains en Europe. Prophétismes, pentecôtismes et politiques des nations, pp. 155-178. Paris : Karthala.

Noret, Joël, 2008. « Mémoire de l'esclavage et capital religieux », Gradhiva 8, pp. 48-63.

Noret, Joël, 2010. « L'invention d'une tradition en question. Mémoire de l'esclavage et construction de l'identité yoruba dans le Bénin méridional », Bulletin des séances de l'Académie royale des sciences d'Outre-Mer 56 (3), pp. 247-264.

Ologoudou, Émile, 2008. «Tours et détours des mémoires familiales à Ouidah : la place de l'esclavage en question », Gradhiva 8 (2), pp 80-86.

Piot, Charles, 1996. « Of slaves and the gift : Kabre Sale of Kin during the era of the slave trade », The Journal of african history 37 (1), pp. 31-49. 
Reis, Joao José \& Guran Milton, 2002. « Urbain-Karim Elisio da Silva, Um Agudà Descendente de Negro Malê », Afro-Ásia 28, pp. 77-96.

Strandsbjerg, Camilla, 2015. Religion et transformations politiques au Bénin. Paris : Karthala.

Stoler, Ann Laure, 2002. Carnal knowledge and imperial power. Race and the intimate in colonial rule. Berkeley, Los Angeles, London : University of California Press.

Tozzo, Émile A. 2004. « Rosine Soglo, famille et entreprise politique », Politique africaine 95 (3), pp. 71-90.

Olinto, Antonio, 1964. Brasileiros na África. Rio de Janeiro : Edições GRD.

Verger, Pierre, 1968. Flux et reflux de la traite des nègres entre le Golfe de Bénin et Bahia de Todos os Santos, du XVIIe au XIXe siècle. Paris \& La Haye : Mouton.

Verger, Pierre, 1992. Os Libertos - Sete Caminhos na Liberdade de Escravos da Bahia no Século XIX. Salvador : Corrupio.

Warnier, Jean-Pierre, 1988. « S. Miers \& I. Kopytoff, eds., Slavery in Africa. Historical and anthropological perspectives. - I. Kopytoff, éd., The African frontier. The reproduction of traditional African societies (compte-rendu) », L'Homme 108, pp. 176-179.

Wimmer, Andreas, 2013. Ethnic boundary making. Institutions, power, networks. Oxford: Oxford University Press.

Zinsou, Emile Derlin \& Luc Zoumènou, 2004. Kojo Tovalou Houénou, précurseur, 1887-1936. Pannégrisme et modernité. Paris : Maisonneuve \& Larose. 